\title{
All in one procedure Versus classical procedure for Hernioplasty in Patients With Inguinal Hernia
}

\section{Raghad Fais Thabet(MBChB) ${ }^{1}$, Mohammed Mohammud Habash $(\mathrm{PhD})^{2}$}

\author{
${ }^{1}$ College of Medicine, University of Diyala, Diyala, Iraq \\ ${ }^{2}$ College of Medicine, University of Diyala, Diyala, Iraq \\ Correspondence Address: \\ Dr. Raghad Fais Thabet \\ College of Medicine, University of Diyala, Baqubah \\ email: raghad89faiz@gmail.com mobile 096407711183643
}

Received: 20 August 2020

Revised: 21 August 2020

Accepted: 20 September 2020

Published: 15 April 2021

Diyala Medical Journal 2021:20(1): 61- 69

\begin{abstract}
Background: Hernia is an unusual bulging of the intra-abdominal viscera through a weak area of the abdominal wall or groin region. Inguinal hernia repair is one of the most frequently performed surgical procedures. In recent years, inguinal hernia (IH) repair was changed from pure tissue repair to prosthetic laparoscopic repair.

Objective: we propose to find a new application for the well-known surgical open mesh inguinal hernia in the treatment of inguinal hernias in adults aiming to improve patients' comfort and to reduce the incidence of chronic neuralgia.

Patients and Methods: . A comparative study was designed to study the outcome of different sizes of mesh used in the hernia operations. In the present study, patients diagnosed with primary unilateral inguinal hernia $(n=130)$ were enrolled from May 2019 to March 2020 at Baquba teaching hospital in Diyala governorate. Enrolled patients were divided into two groups. Group A include patients who have inguinal hernia operated with all in one procedure $(n=65)$ and group $B$ included patients with inguinal hernia operated by the classical method $(n=65)$. Spinal and general anaesthesia were used. Before incision for the patient, ceftriaxone (1gm, intravenously) was administrated over $30 \mathrm{~min}$. The process was accomplished on a one-day surgery basis .According to the devised technique, a new smaller prosthesis was placed on the floor of the inguinal canal in order to strengthen all areas of weakness from which hernias may originate. The mesh was enveloped by a fibrocremasterico sheath avoiding contact with neural structures. Follow-up was carried out at 1 week, 1month, 3months for evaluation of postoperative pain using numbering scale score, need of medication, patients' comfort, and postoperative complications.
\end{abstract}


Results: all patients operated by all in one procedure were discharged within $24 \mathrm{~h}$ from surgery. Slight pain was reported by the majority of patients and $(07.69 \%)$ of them required pain medication at home. After the 1st postoperative week $(95.36 \%)$ reported no pain and $(04.62 \%)$ show bruising genitaliao, $(01.54 \%)$ had seroma and no wound infection recorded. No relevant limitation of normal activities was reported. There have been no postoperative neuralgias. Conclusion: This new hernioplastyo technique respects the anatomy of the inguinal canal, uses a smaller mesh, and seems to avoid neuralgias with maximum comfort for the patients. We recommended to all in one technique o because gives better results and fewer complications.

Keywords: Hernia ;Open mesh inguinal hernia; patients

\section{Introduction}

The word "hernia" means the protrusion of the content of a cavity through an abnormal opening in the wall of the containing cavity because a hernia could occur anywhere in the body and not just in the abdomen. Around 1550BC, the hernia was firstly defined as a disorder in the Mesopotamia and Egyptian cultures [1].

The groin region is associated with the abdominal cavity through which the preperitoneal fat bulge out and it is either congenital or acquired. In some cases, the fetal subsiding of the testicles through the inguinal canal at the abdominal position is known as a congenital hernia [2].

The acquired hernia develops at the later stages of life and it is associated with impaired collagen formation [3]. It leads to develoing severe pain along with lifethreatening complications [4].

After the descent of the fetal testis into the scrotum from the retroperitoneum, the processus virginals should obliterate [5]. If the processus virginals is not obliterated the direct inguinal hernia occurs as a result of the weakening of the posterior wall of the inguinal canal. This region called as "Hesselbach triangle". It is defined medially by the rectus abdominis muscle, laterally by the epigastric vessels, and inferiorly by the inguinal ligament obliterated, fat or bowel may get into it. An indirect inguinal hernia develops as a result of the failure of the processus virginals to fully obliterate. This hernia lies lateral to the inferior epigastric artery (6). The canal has four boundaries [7]. They are as follows:

-Posterior wall - Transversalis fascia laterally; conjoint tendon medially

-Anterior wall - Internal oblique muscle laterally and aponeurosis of external oblique muscle

-Roof - it is internal oblique and transversus abdominis muscles

-Floor - the floor is Inguinal ligament and lacunar ligament (medially)

In males, the inguinal canal consists of the ilioinguinal nerve and spermatic cord. In females, it includes ilioinguinal nerve, a genital branch of the genitofemoral nerve, and a round ligament [8].

Inguinal hernia $(\mathrm{IH})$ repair is one of the most frequently performed surgical procedures. An inguinal hernia does not resolve spontaneously and must be repaired because of the high risk of complications [9]. 
Generally, mesh repair (Lichtenstein tensionfree hernioplasty) and Moloney darn repair were practiced for inguinal hernia repair. Both showed low recurrence rates. Among two, mesh repair was considered to be more recent [10]. Although inguinal hernia repair is the most frequently performed surgical procedure worldwide the ideal repair still not accepted yet [11]. The development of new prosthetic materials and their use in hernia repair surgery has been improved the outcome in many folds [12]. In recent years, IH repair were changed from pure tissue repair to prosthetic laparoscopic repair. Use of fascia and sutures under tension for $\mathrm{IH}$ repair showed a high recurrence of the hernia. Both of these techniques promoted the development of polypropylene mesh to strengthen the posterior wall of the inguinal canal [13].

\section{Patients and Methods}

A comparative study was designed to study the outcome of different sizes of mesh used in the hernia operations. In the present study, patients diagnosed with primary unilateral inguinal hernia $(n=130)$ were enrolled from May 2019 to March 2020 at Baquba teaching hospital in Diyala governorate. Enrolled patients were divided into two groups. Group A includes patients who have inguinal hernia operated with all in one procedure $(n=65)$ and group B included patients with inguinal hernia operated by classical method $(n=65)$. Spinal and general anaesthesia were used.
Before incision for the patient, ceftriaxone (1gm, intravenously) was administrated over $30 \mathrm{~min}$. The process was accomplished on a one-day surgery basis. According to the devised technique, a new smaller prosthesis was placed on the floor of the inguinal canal in order to strengthen all areas of weakness from which hernias may originate. The mesh was enveloped by a fibro-cremasterico sheath avoiding contact with neural structures. Follow-up was carried out at 1week, 1month, 3 months for evaluation of postoperative pain using numbering scale score, the need of medication, patients' comfort and postoperative complications.

\section{Surgical procedure}

Under general and spinal anesthesia in supine position inguinal incision is done. The external oblique muscle fascia was opened and the spermatic cord identification was done medial longitudinal incision the fibrocremasteric sheath (FCS) was done by a diathermocoagulator (figure1) From the medial incision to the inguinal ligament, FCS was exposed. The postero-medial portion of the FCS was dissected by the upward traction on the spermatic cord. It was then left on the transversalis (figure2). Then the hernia sac was separated from the cord elements, excised after ligation and pushed away into the abdominal cavity. Depend on the hernia type, the transversalis fascia is approximated. In the case of direct or indirect hernia, the deep inguinal ring should be prepared. 


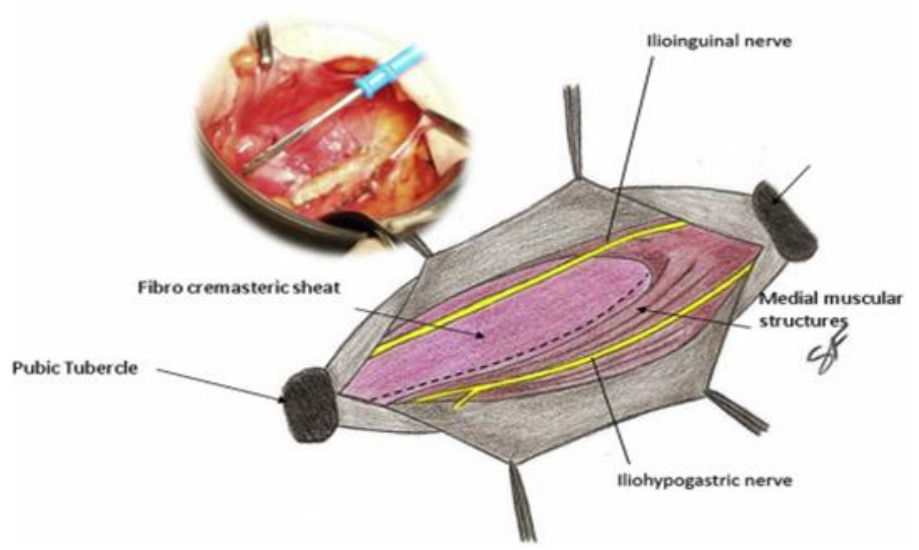

Figure(1): The fibro-cremasteric sheath (FCS) Medial - longitudinal incision

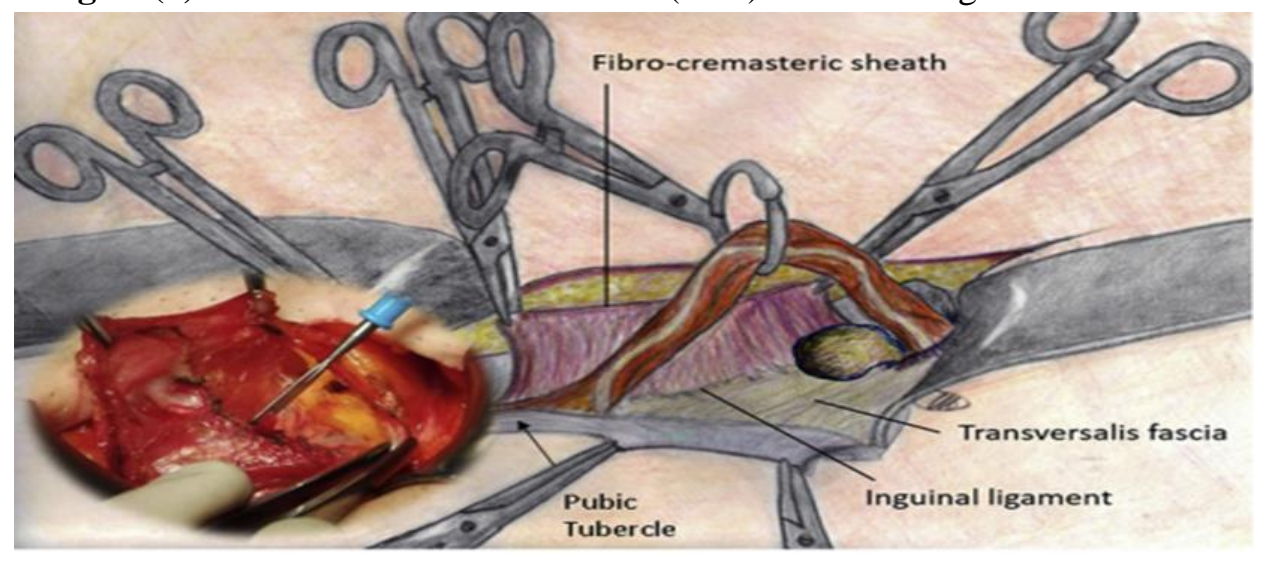

Figure (2): Dissection of fibro-cremasteric sheath (FCS)

A specific shape of mesh was used in the study. The prosthesis consists of 3 sections. These sections are as follows Figure (3).

a) Section A - A ring-shaped portion deliberate to frame the deep inguinal orifice b) Section $\mathrm{B}-\mathrm{A}$ thin connection of the prosthesis between section $\mathrm{A}$ and section $\mathrm{C}$

c) Section C - A trapezoidal shaped part of the mesh which lay on the inguinal canal floor 


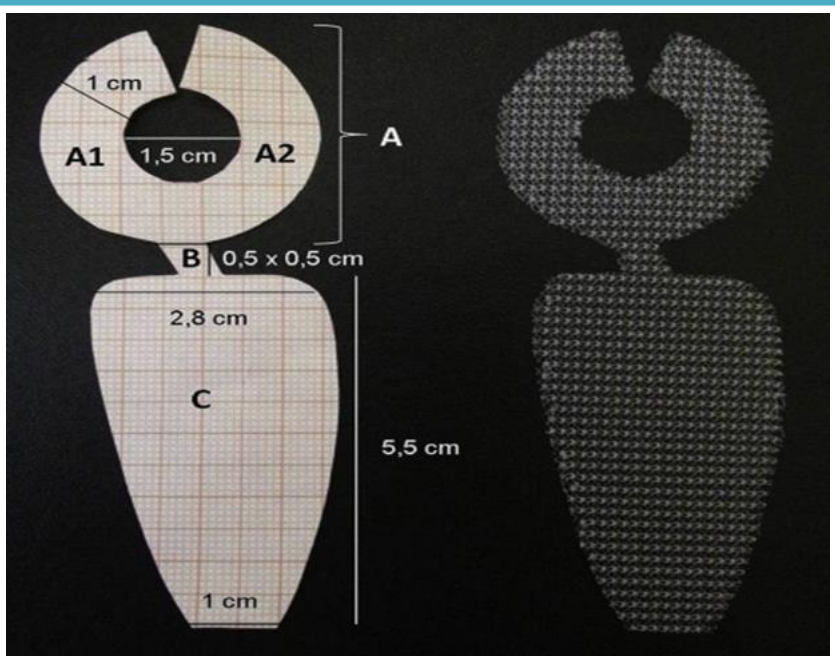

Figure(3): The design of 'all-in-one' mesh

By stitching sections A1 and A2 together, the under the deep inguinal orifice rim to conical ring can be formed. This section A strengthening the lateral weakness area was used to surrounds the spermatic Figure (4). elements. The prosthetic ring was placed

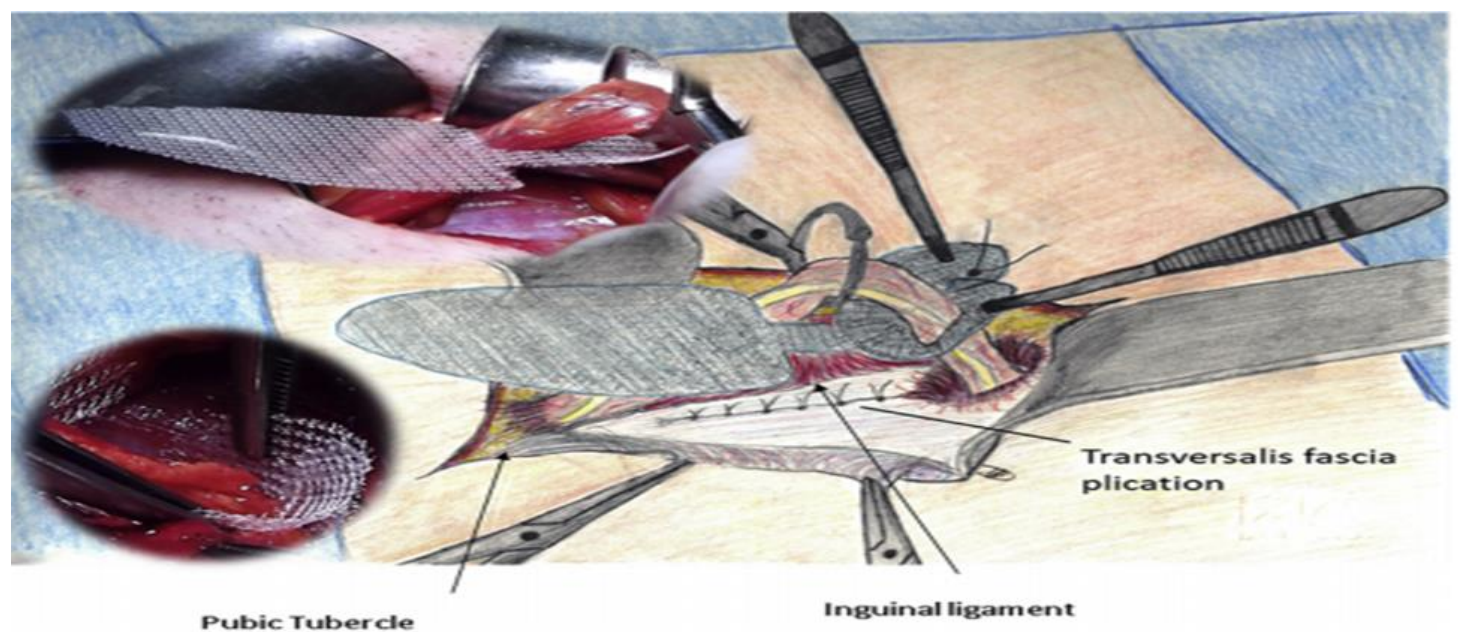

Figure (4): The prosthetic ring (section A) placement in the deep inguinal ring

Above the transversalis fascia, sections B and $\mathrm{C}$ were present. Section $\mathrm{B}$ was present in the form of the ring. It go out to form segment $\mathrm{C}$ with a marginally medial direction. So that, the inguinal canal floor and medially weakens area got exposed for operation. Placed in a specific way, the prosthesis rests crosswise with its lesser convexity on the inguinal ligament and spreads to the conjoined tendon. The prosthesis section $\mathrm{B}$ was under the deep ring which was closed by the absorbable stitches at the lower edge. When the stitch was tightened, the narrowness of section $\mathrm{B}$ evades section $\mathrm{C}$ wrinkling. The section $\mathrm{C}$ tip overlaps the pubic tubercle. It was sutured in place escaping the periosteum. Then the FCS medial margin was recovered and move around underneath the spermatic cord. To cover the mesh, the running absorbable 
suture fixed to the medial muscular structures.

The enveloped prosthesis was halted. It did not require any stitching to adjacent structures. The cremaster grip between spermatic cord and mesh. Finally, the cord was resumed to its normal position and the external oblique muscle aponeurosis was sutured with absorbable material. The prosthesis is fixed from the deep inguinal ring to the pubic tubercle, sandwiched between the cremaster and fascia transversalis. Both left and right-sided hernias can be used due to the shape of the prosthesis.

\section{Statistical analysis}

The significance for different percentages were tested using Chi -square test, Statistical significance was considered whenever $\mathrm{P}$ value was equal or less than 0.05 .

\section{Results}

In the present study, the patient's recovery was assessed in the small size mesh $(n=65)$ and large size $(n=65)$ mesh operated hernia. The postoperative pain was recorded by the surgeon. At the time of discharge, all patients asked for evaluation of postoperative pain, and its quality (mild/severe), postoperative discomfort and medication side effect if any. The patient's discomfort was measured in relation to the limitation of daily activities during the postoperative period, and return to work, and sports. After seven days of postsurgery, a clinical evaluation was made. The follow-up was done for 3 months to assess the outcome of the surgery.

Table (1): Assessment of postoperative pain and analgesia need in the enrolled patients

\begin{tabular}{|c|c|c|c|c|c|}
\hline Parameters & Variable & \multicolumn{2}{|c|}{ Group A } & & oupB 9 \\
\hline \multirow[t]{7}{*}{ Post-operative pain } & Mild & 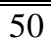 & 76.92 & 49 & 75.39 \\
\hline & Moderate & 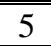 & 07.69 & 15 & 23.08 \\
\hline & Normal & 10 & 15.39 & 1 & 01.54 \\
\hline & $\overline{~ T o t a l}$ & \multicolumn{2}{|c|}{65} & \multicolumn{2}{|r|}{65} \\
\hline & ONNA & 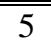 & 07.69 & 25 & 38.46 \\
\hline & No analgesia & 60 & 92.31 & 40 & 61.54 \\
\hline & Total & \multicolumn{2}{|c|}{65} & \multicolumn{2}{|c|}{65} \\
\hline
\end{tabular}

In Table (1) after the operation, the pain was monitored for 1 week. Mild pain was reported by the $50(76.92 \%)$ and $49(75.39 \%)$ patients from the A and B groups, respectively. Moderate pain was reported by the $05(07.69 \%)$ and $15(23.08 \%)$ patients from the A and B groups, respectively. About

$10(15.39 \%)$ and $01(01.54 \%)$ patients reported no post-operative pain from the groups A and B, respectively. Around 05 $(07.69 \%) \quad$ small-size mesh-operated individuals were needed analgesics. Around 25 (38.46\%) large-size mesh-operated individuals were needed analgesics.

Table (2): Observation of post-operative parameters in the A and B group

\begin{tabular}{|l||l||lc||lc||}
\hline \hline Parameters & Variable & Group A \% & Group B $\%$ \\
\hline \hline Urinary retention & Yes & 1 & 01.54 & 10 & 15.39 \\
\hline \hline Bruising genetalia & Yes & 3 & 04.62 & 11 & 16.92 \\
\hline \hline Seroma & Yes & 1 & 01.54 & 13 & 20.00 \\
\hline \hline Wound infection & Yes & 00 & 00.00 & 4 & 06.15 \\
\hline
\end{tabular}


In Table (2) Postoperative parameters such as urinary retardation, bruising genitalia, seroma, and wound infection were recorded. The observation of these parameters has depicted in Table 2. Around $01(01.54 \%)$ and $10(15.39 \%)$ patients showed urinary retardation in the A and B group, respectively. Around 03 (04.62\%) and
11 (16.92\%) patients showed bruising genitalia in the A and B group, respectively. About 01 (01.54\%) and 13 (20.00\%) patients showed seroma after surgery in the A and B groups, respectively. Wound infection was reported by only $04(06.15 \%)$ individuals from group B.

Table (3): Results of patients follow up for 3 months

\begin{tabular}{|c||l||lrr||cc|}
\hline \multicolumn{1}{|c|}{ Parameters } & \multicolumn{1}{|c|}{ Variable } & \multicolumn{2}{|c|}{ Group A } & \multicolumn{2}{c|}{ Group B } & \% \\
\hline \hline \multirow{2}{*}{ 1 week follow up } & Slight pain & 3 & 04.62 & 20 & 30.78 \\
\cline { 2 - 7 } & Normal & 62 & 95.38 & & 45 & 69.23 \\
\hline \multirow{2}{*}{ 1 month follow up } & Slight pain & 00 & 00.00 & 10 & 15.39 \\
\cline { 2 - 7 } & Normal & 65 & 100 & 55 & 84.61 \\
\hline \multirow{2}{*}{3 months follow up } & Slight pain & 00 & 00.00 & 2 & 03.08 \\
\cline { 2 - 7 } & Normal & 65 & 100 & 63 & 96.92 \\
\hline \hline & & \multicolumn{2}{|c|}{65} & \multicolumn{2}{c|}{65} \\
\hline
\end{tabular}

The observations were recorded at $1 \mathrm{st}$ week, 1st month and 3rd months. The data is represented in Table 3 . In the small-size mesh used patient group, 03 (04.62\%) individuals reported having slight pain in the $1 \mathrm{st}$ week. The number of pain reporting individuals gets decreased until the 3rd month. At the end of the study, all the patients showed pain-free activity. Group B also showed a similar trend as group A. In the 1 week, around $20(30.78 \%)$ individuals reported having slight pain. After 1 month, the individuals reported pain was decreased to $10(15.39 \%)$. Small size mesh operated patients showed a maximum recovery rate $(100 \%)$ than the large size mesh operated patients group (96.92\%).

\section{Discussion}

During our study, we faced some difficulties in comparison of our results because we didn't find any study similar to our subject that reported about the (all in one) procedure in Iraq. So we compare our results with studies done about inguinal hernia in general. Postoperative pain is temporary, usually controlled by medication. When persistent after 3 months from surgery, pain becomes disabling and may compromise the patient's quality of life. Pain may be related to the presence of the mesh that, because of size and location, takes contact with muscular structures or caused by fibrotic entrapment of nerves by a subfascial prosthesis $[15,16]$.

Sarhan [17] reported that urinary retention, scrotal swelling and wound infection were the most common complications from hernia surgery. He reported urinary retention $(5.88 \%$ to $9.43 \%)$, scrotal swelling $(11.77 \%$ to $16.98 \%$ ), and wound infection (1.96 to $7.55 \%$ ) in the comparative study of Lichtenstein tension-free hernioplasty and Darn repair. In our study, the rate of wound infection and urinary retention was found to be very low so our study accordance with this. Inguinal hernioplasty (tension-free) is essential for a successful hernia repair. It is 
appropriately fulfilled by both mesh plug repair and a correctly done Shouldice repair [17]. In the present study, small size mesh was found to be more beneficial as compared to large size mesh for hernia repair. Our study was similar to this report. Assim, (16 studied 96 adult patients (20-70 years) with inguinal hernia which repaired by the prolene-mesh as a tension-free Lichtensteinstyle. In this study, only 2 cases were developed seroma. The occurrence of the pain, wound infection and recurrence were very low. Our study is in accordance with this report.

Sadik et al. (2015) reported that the right inguinal hernia $(\mathrm{RIH})$ was more common than the left inguinal hernia (LIH). In our study also, the rate of RIH was found to be more than LIH. Our study is in accordance with this report [18]. In classical operations, hematomas and wound infections are commonly reported. Wound infections and hematomas appearance can be significantly reduced using mesh in the hernia surgery. Mohialdeen et al. (2012) reported hernia operation using the mesh is considerably improved than the ordinal operation due to reduction of recurrence changes, while in the classical operation, recurrence of the disease was generally observed. In the present study, we have been using different sizes of the mesh to operate the hernia in Iraqi patients.

In the inguinal hernia repair can be performed under regional, local or general anaesthesia. For various reasons, inguinal hernia repair under local anaesthesia is not well accepted to both patients and surgeons. The patients fear from pain and surgeons need full relaxation and co-operation to do successful hernia repair [19] studied the hernia surgery under local anesthesia and procedure duration were 30 to $80 \mathrm{~min}$. Further, they reported some cases with superficial wound infection, ecchymosis at the site of infiltration and operation, subcutaneous seroma and scrotal haematoma. However, none of the patients reported having urinary retention, recurrence or mortality. In our study, only one patient showed urinary retention in the SSM so our study in spite of that repair done under GA and spinal anesthesia give alow rate of retention than those done under local anesthesia. Salman, (2011) reported 36 min as the operative time for hernia surgery with mild postoperative pain. The patients were back to normal life after 3-7 days. There is no recurrence and no chronic disabling pain. [20].Our study is in accordance with this report.

\section{Conclusions}

This new hernioplasty technique respects the anatomy of the inguinal canal, uses a smaller mesh, and seems to avoid neuralgia with maximum comfort for the patients.

\section{Recommendations}

We recommended to use a small-size mesh (all in one technique) o because give a better results and less complications.

\section{References}

[1]Sachs M, Damm M, Encke A. Historical evolution of inguinal hernia repair. World $\mathrm{J}$ Surg 1997;21:218-223.

[2]Komorowski AL, Moran Rodriguez J. Amyand's hernia. Historical perspective and current considerations. Acta Chir Belg. 2009 Jul-Aug;109(4):563-4.

[3]Tatay FC. Aproximacion historica al conocimiento de la hernia. Los medicos que 
la describieron $y$ trataron. Hernia inguinocrural. Ethicon, Valencia 2001.

[4]Vasiliadis K, Knaebel H, Djakovic N, et al. Challenging surgical management of a giant inguinoscrotal hernia: Report of a case. Surg Today. 2010;40(7):684-687.

[5]Bax T, Sheppard BC, Crass RA (1999) Surgical Options in the Management of Groin Hernias. Am Fam Physician 59: 14356.

[6]Bingener J, Buck L, Richards M, et al. Long-term outcomes in laparoscopic vs open ventral hernia repair. Arch Surg 2007;142:562-7.

[7]Moore KL, Dalley AF, Agur AMR. Clinically oriented anatomy. 5th ed. Lippincott Williams \& Wilkins: Philadelphia, Pa; 2006. 214-226.

[8]Darke RL, Vogl AW, Mitchell AWM. Gray's anatomy for students. 1st ed. Churchill-Livindstone: Philadelphia, Pa; 2004. 258-265.

[9]Sadik H K, Haithem H A, Haider A J (2015) Pediatric inguinal hernia in basrah. Basrah Journal of Surgery, 21(2): 61-65. [10]Al-Saiegh AM, Al-Saffar RS, AlKhassaki HT (2009) Tension - free inguinal hernia repair comparing 'Mesh' with 'Darn': A prospective randomized clinical trial. Iraqi Academic Scientific Journal, 8(3):220-227 [11]Khazaal AS (2017). Outcome of nonfixation versus fixation of the mesh in Lichtenstein repair of inguinal hernia. The Medical Journal of Tikrit University, 23(1): 25-32.

[12]Assim H (2012) Is it essential to put a drain in patients with Cholecystectomy? Diyala Journal of Medicine, 2(1): 84-90. [13]Salim OA, Al-Sarraf SA, Al-Obaidi SM (2008) Mesh repair versus non mesh repair of primaryinguinal hernia. Journal of the Faculty of Medicine, 50(4): 403-406. [14]Sachs M, Damm M, Encke A. Historical evolution of inguinal hernia repair. World $\mathbf{J}$ Surg. 1997;21(2):218-223.

[15]Al-Saiegh AM, Al-Saffar RS, AlKhassaki HT (2009) Tension - free inguinal hernia repair comparing 'Mesh' with 'Darn': A prospective randomized clinical trial. Iraqi Academic Scientific Journal, 8(3):220-227. [16]Assim H (2012) Is it essential to put a drain in patients with Cholecystectomy? Diyala Journal of Medicine, 2(1): 84-90. [17]Sarhan HH (2008) Tension-free inguinal hernia repair comparing shouldice repair with mesh plug repair: A prospective randomized controlled clinical trial. The Medical Journal of Tikrit University, 2(142): 145-151.

[18]Salman IA, Jwad AM (2017). Iraqi Academic Scientific Journal, 16(3): 248-251. [19]Sanders DL, Kingsnorth AN. Prosthetic mesh materials used in hernia surgery. Expert Rev Med Devices. 2012;9(2):159-179. [20]Aufenacker TJ, Schmits SP, Gouma DJ, Simons MP. Do guidelines influence results in inguinal hernia treatment? A descriptive study of 2,535 hernia repairs in one teaching hospital from 1994 to 2004. Hernia. 2009;13(1):35-39. 